\title{
Dictynna
}

Dictynna

Revue de poétique latine

$1 \mid 2004$

Varia

\section{Tibulle, 1,4 : L'élégie et la tradition poétique du discours didactique}

\section{Jacqueline Fabre-Serris}

\section{(2) OpenEdition \\ 1 Journals}

Édition électronique

URL : http://journals.openedition.org/dictynna/162

DOI : 10.4000/dictynna.162

ISSN : $1765-3142$

Édition imprimée

ISBN : 2-84467-088-1

Référence électronique

Jacqueline Fabre-Serris, "Tibulle, 1,4 : L'élégie et la tradition poétique du discours didactique », Dictynna [En ligne], 1 | 2004, mis en ligne le 15 novembre 2010, consulté le 11 septembre 2020. URL : http://journals.openedition.org/dictynna/162 ; DOI : https://doi.org/10.4000/dictynna.162

Ce document a été généré automatiquement le 11 septembre 2020.

\section{(c) (i) () $९$}

Les contenus des la revue Dictynna sont mis à disposition selon les termes de la Licence Creative Commons Attribution - Pas d'Utilisation Commerciale - Pas de Modification 4.0 International. 


\title{
Tibulle, $1,4:$ L'élégie et la tradition poétique du discours didactique
}

\author{
Jacqueline Fabre-Serris
}

1 Le goût de l'ingenium, souvent reproché à Ovide, est un trait qu'il partage avec Tibulle. L'élégie 1,4 de ce dernier, modèle de l'Art d'aimer, est tout à fait typique de la façon dont écrit le successeur de Gallus ${ }^{1}$ : c'est un texte qui ne cesse de chercher l'effet de surprise, et ce, dès les premiers vers, avec le choix de la forme du dialogue comme cadre à un exposé didactique sur l'amour.

2 L'élégie érotique romaine est un genre littéraire qui se caractérise par l'adoption d'une situation particulière d'énonciation: l'auteur utilise le «je» pour faire part d'une expérience de l'amour et de la poésie présentée comme personnelle. Dans l'élégie 1,4 cette règle est modifiée $:$ le « je » est mis en scène dans une relation de dialogue avec un dieu, qu'il interroge sur son habileté dans le domaine amoureux ; la divinité répond par un long exposé, qui développe, en fait, la position élégiaque sur le sujet, exposé qui est suivi d'une reprise de parole, et, avec elle, du retour à la perspective habituelle de l'élégie.

3 L'ingéniosité, chez Tibulle, est un élément déterminant de son rapport au lecteur ; c'est une façon d'éveiller son intérêt et de stimuler sa réflexion. Ici le renoncement inattendu à une règle quasiment constitutive de l'élégie engage implicitement ce dernier à se demander pourquoi le poète a recouru aux marques formelles du genre didactique pour traiter de l'amour selon la thématique élégiaque.

4 L'alternance questions-réponses a un précédent hellénistique fameux : c'est ainsi que Callimaque procède, semble-t-il, dans les deux premiers livres des Aitia, où il se met en scène en compagnie des Muses, qui répondent à ses demandes successives sur divers rites plus ou moins obscurs.

5 À Rome, la tradition du discours didactique proprement dit, est, à l'époque de Tibulle, illustrée par deux grands textes: le De rerum natura et les Géorgiques, dont le premier n'est pas sans avoir fortement influencé le second. Ces deux textes prônent, chacun, un mode de vie qui exclut les passions et plus particulièrement l'amour. Lucrèce conclut 
l'un de ses livres sur une condamnation de la passion amoureuse ; Virgile termine son poème sur un épisode qui dénonce le furor érotique.

6 L'insertion d'un certain nombre de références intertextuelles dans ses textes est, après la volonté de surprendre, le second dispositif utilisé par Tibulle pour orienter l'interprétation de ses lecteurs. Dans l'élégie 1,4, elles consistent principalement en renvois à Lucrèce, à Virgile et au fondateur du genre et de la tradition élégiaques, Gallus.

7 Je voudrais montrer ici comment Tibulle a joué, en poète doctus, sur la culture et la complicité intellectuelle d'un lecteur aussi savant que lui et, plus particulièrement, comment il s'est servi de certains motifs ou textes relevant soit du poème didactique, soit de l'élégie ou de ses modèles hellénistiques pour rédiger une espèce de manifeste en faveur du genre qu'il pratique. Son poème propose en effet une rencontre entre élégie et poésie didactique, qui est conçue comme une réponse au traitement polémique des motifs érotiques et élégiaques auquel s'étaient livrés Lucrèce et Virgile. En l'occurrence, la démarche suivie a été, dès son point de départ, extrêmement subtile: Tibulle - ce sera l'hypothèse que ce papier va tenter de fonder - a, pour défendre son exposé de la conception élégiaque de la poésie et de l'existence, recouru à un motif célèbre de la tradition érotique hellénistique, que Virgile avait déjà utilisé dans le cadre des Géorgiques, mais en le détournant à ses propres fins, pour donner plus de force à l'idéal de vie du paysan qu'il avait choisi de célébrer dans son poème didactique.

8 Le De rerum natura et les Géorgiques ont visé à modifier en profondeur la vision du monde, voire le mode de vie de leurs lecteurs. Chez Lucrèce, comme chez Virgile, l'amour est une passion à proscrire. Le premier se livre à une analyse de ses ravages; le second choisit la voie de la fiction mythique. Il évoque avec l'histoire du poète Orphée, un exemple de vie détruite par la passion amoureuse et, pour que la leçon soit plus claire, travaille le cadre narratif dans lequel il l'insère : l'histoire d'Orphée fait partie de celle d'Aristée, qui illustre un autre genre de vie, celui du paysan dominé, lui, par le labor et la pietas².

9 Cet ensemble narratif est précédé par un texte qui a été souvent interprété comme l'écho d'une rencontre personnelle et qui évoque le labor fructueux d'un vieillard vu dans la campagne de Tarente. Il s'agit d'une description d'un choix de vie, qui est, en quelque sorte, l'équivalent de celui d'Aristée. Si l'on considère que le labor et la pietas sont les deux grandes valeurs des Géorgiques, il y une espèce de répartition de ces valeurs dans les deux épisodes : dans la description de l'existence menée par le vieillard corycien, l'accent est mis sur le labor, dans le récit qui a pour héros Aristée, il est mis sur la pietas (l'apiculteur fait seulement allusion à un labor brutalement et inexplicablement anéanti).

10 Dans un article publié en 1992 dans M.D., R. Thomas a proposé de l'épisode du vieillard de Tarente une analyse très suggestive ${ }^{3}$. Il part de l'article d'E. Bowie sur «l'idylle 7 de Théocrite, Philétas et Longus $»^{4}$ qu'il complète par la monographie que Du Quesnay a consacrée à la Bucolique $1^{5}$. Je renvoie, sans entrer dans le détail complexe de leurs argumentations, à ces articles qui proposent l'hypothèse que Philétas ait composé de la poésie pastorale et qu'il soit à l'origine de certains motifs de la poésie grecque et latine.

11 Ce qui a intéressé plus particulièrement $R$. Thomas, c'est l'un de ces motifs imputables à Philétas : le personnage du vieil homme, dont on aurait des avatars dans le Lycidas de Théocrite, dans le Tityre de Virgile, dans le Philétas de Longus et des échos dans la 
poésie élégiaque (ainsi dans le vers 30 de l'élégie 2, 34 de Properce : nil iuuat in magno uester amore senex).

Au terme d'une confrontation détaillée du texte des Géorgiques avec des passages de Longus, R. Thomas propose de considérer que le vieillard de Tarente, possesseur d'un jardin qu'il cultive à longueur d'année, est, non pas le souvenir d'une rencontre, mais une pure création virgilienne qui renverrait à la figure de vieillard rendue célèbre par Philétas, et dont on peut supposer, à partir de fragments de Philétas lui-même et du texte de Longus, qu'elle était aussi associée à la possession d'un jardin.

Pour ma part, je retiendrai ici de l'article très riche de R. Thomas le fait que, chez Théocrite et chez Longus, ce vieillard a une compétence reconnue dans le domaine amoureux, que l'on peut donc supposer qu'il en était de même chez l'original (d'autant que Philétas est un modèle revendiqué par Properce) et, que sur ce point, Virgile a opéré, une complète révolution au livre 4 des Géorgiques. Un autre texte peut être ajouté à la série étudiée par $\mathrm{R}$. Thomas : il s'agit du fragment 10 des Bucoliques de Bion, où un jeune oiseleur, qui tente - en vain - de capturer Eros, qu'il prend pour un oiseau, demande conseil à un vieux laboureur qui lui a enseigné cet art ; ce dernier lui répond de renoncer à ce gibier qui viendra de lui-même se poser sur sa tête, quand il aura atteint l'âge d'homme. La scène fait penser au discours du Philétas de Longus, qui raconte s'être épuisé à attraper un enfant qui allait de branche en branche et dans lequel il a fini par reconnaître Eros.

Le mode de vie du vieillard de Tarente est, comme je l'ai dit plus haut, une leçon en actes : le terrain dont il a hérité est pauvre; mais à force de curae et de labor, il égale animis (dans l'esprit du paysan qui le cultive) ses richesses à celles des rois. Le remplacement du discours didactique par une simple description participe, me semblet-il, du passage au second plan dans les Géorgiques de la figure du poète au profit de celle du paysan, dont l'existence, régie par les valeurs du labor et de la pietas, est célébrée comme l'idéal de vie par excellence. On a, dans cet épisode, une anticipation de l'histoire finale, qui oppose au mode de vie de l'apiculteur Aristée celui du poète Orphée, dans lequel - je suis, sur ce point, les analyses de G. Conte on peut voir une allusion au poète élégiaque Gallus, dont le choix de vie et de poésie était déjà contesté dans la Bucolique 10.

Je propose - en revenant à l'objet de mon article de voir dans l'élégie 1,4, qui met en scène, Priape ${ }^{6}$, le dieu des jardins, en situation de praeceptor amoris et qui fait allusion à une compétence que Tibulle aura acquise en matière de Veneris praecepta une fois devenu senex, une réponse à l'épisode du vieillard de Tarente. Certes on ne trouve, chez Tibulle, aucune allusion à l'âge du dieu; mais il en est de même dans les Carmina Priapea, à l'exception du carmen 76, où Priape se présente comme un vieillard : quod sim iam senior meumque canis/cum barba caput albicet capillis (v. 1-2) ${ }^{7}$.

Je voudrais essayer de montrer que le discours amoureux prononcé par Priape est une théorisation de l'élégie telle qu'elle avait été - thématiquement - constituée par Gallus. En d'autres termes, il s'est agi pour Tibulle de prouver que l'élégie pouvait être tout autre chose que les plaintes, pleurs et ressassements du nom de la bien-aimée auxquels la réduit Virgile dans la version qu'il donne dans les Géorgiques de la poésie d'Orphée. La description du domaine du vieillard de Tarente se présente comme une digression rattachée à un passage sur les jardins qui est, lui, en relation avec le sujet du livre 4 : les abeilles. Heureux hasard s'il en est ou incitation indirecte, ce passage s'ouvre sur une évocation du dieu protecteur de ces jardins : Priape. 
17 C'est pour sa compétence dans l'art de séduire les garçons que ce même dieu est choisi par Tibulle dans l'élégie 1,4. Priape est un personnage divin fortement connoté sexuellement et parodiquement. Il est qualifié ici de Bacchi rustica proles (v. 7). L'invocation qui lui est adressée souligne cet aspect rustique, qui contraste avec l'habileté que l'amant-poète lui prête : il n'y a aucun cultus dans sa barbe et dans sa chevelure, deux éléments traditionnels de la beauté masculine; et son corps est nu.

L'apparition de Priape dans un contexte amoureux n'est pas sans antécédent: dans l'idylle 1 de Théocrite, il fait partie des dieux qui viennent voir Daphnis, consumé par une passion à laquelle il ne veut pas céder. Le dieu reproche au chevrier d'être à la fois

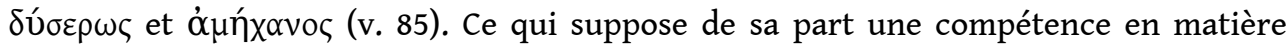
d'amour supérieure à celle de Daphnis. Le fait que Virgile ait revendiqué pour modèle Théocrite a probablement compté dans le choix de Tibulle, dont l'élégie renvoie peutêtre aussi à l'épigramme $4 \mathrm{du}$ même Théocrite. Selon F. Cairns ${ }^{8}$, on aurait en effet dans la situation imaginée par Tibulle un écho de la mise en scène décrite dans cette épigramme, où le poète charge un chevrier de solliciter pour lui l'aide de Priape dans une affaire amoureuse.

19 Les exemples de rustici, auteurs de poésie amoureuse, sont, par ailleurs, nombreux dans la littérature pastorale: il n'est que de citer le Cyclope chez Théocrite et chez Callimaque, Pan, Silène et Corydon chez Virgile. Cette tradition remonte probablement au Banquet de Platon, où Alcibiade compare Socrate à un Silène ou au satyre Marsyas au moment même où il reconnaît l'exceptionnel pouvoir de ses discours.

20 À Rome, Priape est un personnage récurrent dans une série de textes poétiques, que l'on a appelés de son nom : les Carmina Priapea ${ }^{9}$, dont les auteurs nous sont inconnus et que l'on date, de façon imprécise, entre la première moitié du $\mathrm{I}^{\mathrm{er}}$ siècle av. J.-C. et la fin du I ${ }^{\text {er }}$ siècle ap. J.-C. ${ }^{10}$ Dans ces textes, présentés comme des inscriptions accrochées à la statue du dieu, aisément identifiable à son sexe dressé, Priape, chargé de protéger dans les jardins fruits et légumes des oiseaux et des voleurs, menace les éventuels chapardeurs de divers outrages sexuels ou monnaye leurs faveurs en échange de quelques fruits. Il fait souvent allusion à son sexe exhibé et aux désirs qu'il éprouve ou qu'il suscite. Le contenu de ses discours est crûment érotique ; il n'est question que de satisfactions immédiates.

21 On est ici très loin de la rhétorique et de la stratégie de la patience que développe le Priape tibulléen. Aussi évoquer, à propos des antécédents de l'élégie 1,4, la tradition latine des carmina Priapea ne me semble pertinent que comme signe de l'importance acquise par le dieu dans la culture romaine. La place prise par Priape au I ${ }^{\mathrm{er}}$ siècle av. J.C. dans la littérature romaine (il est présent chez les neoteroi : chez Catulle, chez Marcus Furius Bibaculus et probablement chez Valerius Cato) a pu aussi inciter Tibulle à choisir le dieu comme porte-parole au moment où il cherchait à se référer à la tradition grecque des discoureurs d'amour en apparence rustres et grotesques, que j'ai déjà évoquée, tradition qui - dans un de ses textes les plus importants, l'idylle 1 de Théocrite - incluait, du reste, Priape. Le choix d'un tel porte-parole s'accorde, en outre, avec une orientation thématique propre aux élégies de Tibulle: c'est de tous les élégiaques celui qui dans ses poèmes accorde la place la plus importante au rus auquel il associe des amours heureuses. À cet égard, son Priape, dieu des jardins et maître dans l'art d'aimer, a un correspondant dans le Cupidon de l'élégie 2,1 né, aux commencements des temps, dans les champs parmi les cavales. 
Venons-en maintenant aux propos du dieu. Il faut, dit Priape, fuir la compagnie des jeunes garçons, qui fait toujours naître un iustus amor (v. 10). Ce premier conseil est apparemment - paradoxal, car, s'il était suivi, il annulerait tous les autres. Il entre dans cette stratégie de la surprise qui caractérise la poésie de Tibulle. C'est aussi une façon habile - de commencer le discours par une justification de son objet.

Le second conseil est moins surprenant: si le garçon oppose un refus, le dieu engage son interlocuteur à ne pas être saisi par un sentiment d'impuissance et de lassitude mêlées : le taedium, qui se traduirait par une inaction. Car le temps produit de lui-même des effets: Priape donne pour exemples le domptage des animaux, la dissolution de la pierre minée par l'eau, le mûrissement des raisons et le retour des astres.

Le motif d'une action invisible dont le temps finit par révéler les résultats est emprunté au livre 1 du De rerum natura, ainsi qu'un des exemples évoqués : celui du rocher creusé par l'eau (v. 313). Plusieurs des mots employés dans ce passage : paulatim (v. 15 et De rer. nat., 1, 323), docuit (v. 17, terme récurrent dans le De rerum natura), saxa peredit (v. 18), qui est une transcription de l'expression saxa peresa (De rer. nat., I, 326), ou encore lucida signa (v. 20 et De rer. nat., 5, 518) visent aussi à lui donner une tonalité lucrétienne.

Lucrèce est un des auteurs latins qui a le plus influencé Virgile. C'est à ce dernier que renvoient les autres exemples: l'adoucissement de la sauvagerie est un des bienfaits accomplis par Daphnis dans la Bucolique 5, la mise sous le joug des bœufs et la culture de la vigne appartiennent à la thématique des Géorgiques. L'expression in apricis (...) collibus est reprise du vers 49 de la Bucolique 9.

Cette double coloration lucrétienne et virgilienne donnée d'entrée au discours de Priape justifie, en même temps qu'il authentifie, sa forme didactique : l'amour obéit à la même loi temporelle que les réalités naturelles et les activités humaines qui forment la matière du poème virgilien. À ce titre, il peut faire partie des artes apparus en même temps que l'improbus labor (Géorg., 1, 145-146). Ajoutons que Lucrèce avait également recouru à l'exemple de la goutte d'eau creusant les rochers à propos des effets de l'habitude en amour, immédiatement après une notation sur la façon de faire naître un tel sentiment: nam facit ipsa suis interdum femina factis/morigerisque modis et munde corpore culto,/ut facile insuescat(te) secum degere uitam (4, 1280-1282). Autrement dit, dans un contexte proche par le sens de l'ars amandi que se met à développer Priape.

Le troisième conseil donné par le dieu est de ne pas craindre d'exprimer son amour par des serments. On a là une variante - fréquente - de la proclamation d'amour qui est essentielle dans la thématique élégiaque, où l'amant s'oppose à ses rivaux en faisant fond sur la force de ses sentiments. Priape rassure son interlocuteur : le respect de ces serments-là n'est pas requis et en cas de parjure il n'a rien à craindre des dieux. Ce motif est développé par divers exemples pris dans la nature dont l'un, celui du cheval d'Elide, renvoie sans doute à Virgile (Géorg., 2, 179 et suiv.)

L'ensemble constitue un groupe relativement important de 12 vers dont F. Cairns, qui a étudié l'ordonnance du discours de Priape, remarque qu'ils se situent au centre du texte : les autres motifs se répartissent autour de ce noyau, en se répondant deux par deux selon une structure d'embrassement (ce qu'il appelle une ring-composition) ${ }^{11}$. Il remarque qu'on a ici un développement inattendu, car le motif est traité, non pas, comme c'est le cas habituellement du point de vue de l'aimé, mais du point de vue de l'amant. C'est ce dernier qui est invité à tirer les conséquences de la loi naturelle du 
vieillissement, qui va atteindre l'objet de son désir et le rendre moins attractif. Peutêtre a-t-on là un nouvel écho de Théocrite, qui, dans l'idylle 7, use du motif de la beauté éphémère pour guérir un amant malheureux de sa passion pour un garçon qui le dédaigne.

C'est toutefois avec le conseil suivant, que Priape entre dans le vif du sujet. Il propose une série de comportements qui relèvent tous de l'obsequium: Tu, puero quodcumque tuo temptare libebit,/cedas : obsequio plurima uincet amor (v. 39-40). Vaincre en cédant, tel est donc le secret de la sollertia de Priape. C'est aussi le précepte central de la poétique gallienne, retranscrite ici jusque dans le choix de la formulation: on a avec le mot obsequium un synonyme de seruitium et avec uincet amor une variante ou une citation d'une expression gallienne dont on trouve de multiples équivalents dans la poésie augustéenne ${ }^{12}$.

31 Priape détaille ensuite ces " preuves d'amour » en actes. Elles consistent à accompagner le puer aimé partout où il le souhaite en supportant les épreuves qui se présentent. Il faut donc être à ses côtés en voyage sur terre (malgré la longueur de la route, l'excès de la chaleur ou la menace d'un orage) ou sur mer (même s'il faut manier la rame) ou à la chasse (en acceptant de placer les pièges ou de porter les filets) ou encore engager avec lui, s'il le désire, une lutte en en le laissant gagner.

L'une des activités énumérées par Priape semble avoir eu une place à part dans la poésie gallienne : la chasse, évoquée par l'auteur des Amores à propos de Milanion, qui accepta d'y accompagner la belle Atalante et finit par vaincre sa résistance. C'est aussi sur la notation d'une victoire que se termine la liste de ces divers obsequia: en luttant contre le puer, on peut tenter de lui ravir des baisers et, s'il résiste au début, il finira par les accorder et même par les réclamer ${ }^{13}$.

Le développement suivant surprend dans un premier temps : il porte sur la réception de la poésie et s'adresse non plus à l'interlocuteur de Priape, mais aux pueri, que le dieu avertit: Pieridas, pueri, doctos et amate poetas,/aurea nec superent munera Pieridas (v. 61-62). En fait, il n'est pas incongru de trouver un passage sur la poésie après la liste des conseils pratiques donnés par Priape. Car, en tant que moyen privilégié par le poète pour proclamer son amour, la poésie relève du seruitium amoris.

On trouve une juxtaposition du même genre dans la Bucolique 10, où Virgile propose à Gallus de moduler ses propres motifs dans le cadre du genre bucolique et non plus dans celui du genre élégiaque. Deux actes sont évoqués alors par Gallus. L'un, c'est graver ses Amores sur l'écorce des arbres, un geste qui réitère celui d'Acontius inscrivant le nom de Cydippé sur l'écorce des arbres, mais qui peut aussi être compris comme une façon de désigner l'activité poétique, puisque le terme Amores est également le titre du recueil de Gallus. L'autre, c'est chasser en compagnie des nymphes (pour oublier Cythéris, et non pas comme Milanion avec Atalante pour la conquérir) ${ }^{14}$.

Dans ces conditions, il n'est pas étonnant de trouver chez Tibulle après une énumération de techniques de séduction relevant de l'obsequium amoris, toute une réflexion sur la poésie en tant qu'ars, auquel les pueri préfèrent aujourd'hui les munera. L'idée que le statut social de la poésie s'est dégradé au fil des siècles rappelle le passage du livre $5 \mathrm{du}$ De rerum natura, où Lucrèce raconte l'invention de la poésie. C'est un texte qui a manifestement influencé Tibulle, car on en trouve des échos dans l'élégie 2,3. Le philosophe y observe que le plaisir goûté alors à l'art de la flûte était lié à la nouveauté de sa découverte, que l'homme a continué de s'engouer de tout ce qui est nouveau et lui 
paraît meilleur et que c'est là ce qui explique son actuelle passion pour la pourpre et l'or (deux exemples souvent cités de présents corrupteurs).

Le discours de Priape prend alors l'allure d'une exhortation adressée aux pueri sommés de changer de comportement et de préférer la poésie aux munera. Cette adjuration qui vise à restituer à la poésie en tant qu'ars son rang de valeur originelle s'accompagne de toute une argumentation.

Le point de départ est une affirmation d'ordre général : la poésie a inventé les mythes (Priape prend l'exemple du cheveu de pourpre de Nisus et de l'épaule d'ivoire de Pélops). On peut en conclure qu'elle est une garantie d'éternité : quem referent Musae uiuet (v. 65). Celui qui, à l'inverse, sera insensible aux Muses et monnayera son amour, connaîtra, dès à présent, un sort misérable : il sera séduit par le culte de Cybèle dont les sectateurs se castrent, et puni donc par où il a péché. On a là un exemple du motif fréquent dans la poésie hellénistique : celui de la punition d'un insensible par une divinité qui prend les parts d'un amant méprisé. D’où une conclusion sur ce que veut Vénus : ... querellis/supplicibus, miseris fletibus illa fauet (v. 70-72).

Avec les querellae et les fletus, on retrouve des termes spécifiques du genre élégiaque, après un passage général sur la poésie où les mots Piérides et Muses sont, chacun, répétés deux fois, ce qui est une façon de donner de la solennité à une poésie de genre mineur comme l'élégie, mais sans doute aussi un héritage de la poésie gallienne si l'on se réfère à un des vers du papyrus de Qasr Ibrim (tandem fecerunt c(ar)mina Musae/quae possem domina deicere digna mea).

39 Ainsi finit le discours de Priape. Mais la suite du texte est essentielle, car il y est précisément question de la réception de cette poésie. Le lecteur va assister alors à une série de transformations de la situation d'énonciation.

La première touche le destinataire : on passe du «je » qui a questionné Priape à un certain Titius, présenté comme celui à qui le dieu souhaite que son discours soit transmis. F. Cairns ${ }^{15}$ a proposé d'identifier ce Titius à M. Titius, qui avait été, comme Messala, un partisan d'Antoine avant de se rallier à Octave et dont le mariage avec Fabia Paullina était peut-être récent. Pour justifier la présence d'une allusion à ce personnage, il avance l'hypothèse d'une intervention - pour des raisons d'alliance politique - de Messala. Si on le suit sur ce point, on aurait là une justification de ce statut de choix extérieur, attribué à un dieu.

41 Titius est ensuite récusé comme destinataire par le "je », car il vient de se marier et n'est plus dans la situation d'un amant en quête d'artes pour conquérir les faveurs d'un puer: son épouse lui défend de continuer à s'intéresser aux beaux garçons. Du coup, tout est changé : le «je » prend la place de Priape (instruit par lui, il est devenu un magister, reconnu comme tel (uos me celebrate magistrum, v. 75), apte à se mesurer à un puer lui aussi callidus arte.

42 Ce nouveau praeceptor amoris définit ensuite ceux qui seront dans un futur proche ses propres destinataires : qui spernentur amantes (on retrouve là le public des élégiaques), et laisse entendre que la maitrise des artes lui permettra une fois devenu vieux, de se retrouver entouré d'une troupe de garçons (turba), sans doute tenera comme le disait Priape au début de son discours, mais cette fois - aussi - sedula.

Mais tout cela n'est que projection dans l'avenir: un ultime retournement établit comme destinataire immédiat Marathus, le puer aimé, face à qui le « je «s'avoue à court d'artes: deficiunt artes, deficiunt doli (v. 82). L'élégie 1,4 a, sans cesse, fait alterner deux 
types de rapports : celui de l'amant avec le puer dont il est épris ; et celui du poète avec son lecteur sous la variante du praeceptor amoris avec un ou des interlocuteurs. D'où une fin qui conjoint les deux rapports : parce, puer, quaeso, ne turpis fabula fiam,/cum mea ridebunt uana magisteria (v. 83-84). S'il a été attentif à la façon dont Tibulle lance et reprend les motifs, le lecteur n'est pas invité ici à mettre en doute l'efficacité de l'ars amatoria qui vient d'être présentée ; le finale du discours de Priape portait précisément sur les plaintes et pleurs, dont Vénus serait l'alliée. Cet apparent aveu de faiblesse n'est en fait qu'un ultime Veneris praeceptum!

En ce sens, la prière finale adressée à Marathus n'affaiblit pas la défense de l'élégie qui a été élaborée. En détaillant la poétique gallienne sous la forme de conseils didactiques, Tibulle vient de mettre en évidence d'une part que sa finalité est la conquête de l'être aimé, d'autre part qu'il est tout à fait possible d'associer au mode de vie du poète amoureux la pratique des artes, la patience et le labor. Lesquelles sont aussi trois caractéristiques de la vie des paysans, que les Géorgiques avaient célébrée comme une des plus hautes réalisations de l'existence humaine. Le choix de terminer sur une querella est à interpréter dans la même perspective comme une façon de ne pas se dérober à la critique virgilienne de l'élégie, qui réduisait les chants d'Orphée à l'expression de la plainte amoureuse. La réponse de Tibulle est double: l'élégie est évidemment aussi querella, mais, quand elle l'est, il ne s'agit que d'une de ses stratégies, la dernière, et non la moins efficace, car elle a de son côté Vénus, déesse puissante s'il en est ${ }^{16}$.

Le texte de Tibulle marque incontestablement une étape dans la maturation du genre. Cette entrée de l'élégie dans la tradition du discours didactique montre que l'on en est désormais au stade de la réflexion sur ce qu'est ce nouveau genre. Le temps est venu, sous la pression des prises de position virgiliennes, de développer la poétique gallienne en ars. L'habileté de Tibulle est de le faire en se réclamant de la tradition de la poésie érotique hellénistique dont Virgile s'était auparavant démarqué dans les Géorgiques, et qui est précisément celle dans laquelle s'inscrit l'élégie romaine.

Avec le rustique Priape, dieu des jardins, Tibulle reprend le motif du vieil homme de la campagne devenu un expert en amour, qui remonte, semble-t-il, à Philétas et dont on trouve des échos chez le Lycidas de Théocrite et chez le vieux laboureur de Bion. Il conforte ainsi l'élégie en rappelant la tradition dont elle est l'héritière tout en répondant à Virgile, qui venait de proposer un contre-modèle de ce motif hellénistique avec le vieillard de Tarente.

Dans son article, $R$. Thomas avance une hypothèse très séduisante à propos de la localisation inattendue que Properce donne aux chants de Tityre dans l'élégie 2,34 quand il parle des pins qui ombragent le Galèse. Ce cours d'eau est associé, en réalité, par Virgile au vieillard de Tarente. Selon R. Thomas, on aurait ici, chez Properce, une façon polémique de réaffirmer le caractère originel de la figure du praeceptor amoris ${ }^{17}$. Cette suggestion pourrait, me semble-t-il, être confortée par la possibilité que l'élégie 1,4 ait été un relais entre les textes de Virgile et de Properce.

Incarner cette figure dans Priape, qui était devenu le porte-parole d'une série d'épigrammes érotiques parodiques, permet en même temps à Tibulle de souligner une dimension particulière de l'élégie: la posture ironique, qui sera particulièrement développée chez Properce et Ovide. Lucrèce et Virgile donnent de l'amour une vision tragique ; même quand ils en souffrent, les élégiaques savent se mettre dans la position de commentateurs lucides de ce que Tibulle appelle une fabula turpis. Avec l'Art d'aimer 
d'Ovide, qui, en héritier de Tibulle, assumera, lui-même, la position de magister amoris, cette ironie évoluera vers le cynisme ${ }^{18}$.

\section{NOTES}

1. Je ne suis convaincue par les arguments qu'avance $P$. Murgatroyd, pour défendre son hypothèse de l'antériorité du monobiblos par rapport au livre I des élégies de Tibulle (Tibullus I, A Commentary on the First Book of the Elegies of Albius Tibullus, Pietermaritzburg, 1980, p. 13-15). Dans les Tristes, qui font référence à certaines élégies du livre 1, Tibulle est situé par Ovide entre Gallus et Properce. L'élégie 1,1 de Properce me semble, en particulier, avoir été écrite après l'élégie 1,4 de Tibulle.

2. Je renvoie aux études de G. Conte : Aristeo, Orfeo e le Georgiche. Structura narrativa e funzione didascalica di un mito in Virgilio, il genere e i suoi confini, Milano, 1984, pp. 43-53; Aristeo, Orfeo e le Georgiche. Una seconda volta, « S.C.O. », 1998, pp. 103-128.

3. R. Thomas, The Old Man Revisited. Memory, Reference and Genre in Virg., Georg., 4, 116-148, «M.D. ", 1992, p. 35-70.

4. E.L. Bowie, Theocritus'seventh Idyll, Philitas and Longus, « Class. Quart. », 1985, p. 67-91.

5. I.M. Le M. Du Quesnay, Vergil's First Eclogue, «Papers of the Liverpool Latin Seminar », 1981, p. 30-182.

6. Sur Priape, voir le répertoire de W.-R. Megow dans Lex. Iconogr. Myth. Class. (Suppl.), VIII.1 pp. 1028-1044 et VIII.2 pp. 680-694.

7. Sur le type du vieux Priape barbu, voir Herter, De Priapo in « Religionsgeschichtliche Versuche und Vorarbeiten ", 1932 pp. 182 et suiv.

8. F. Cairns, Tibullus, A Hellenistic Poet at Rome, Cambridge, 1979, p. 190.

9. Voir C. Goldberg, Carmina Priapea. Einleitung, Übersetzung, Interpretation und Kommentar, Heidelberg, 1992.

10. P. E. M. O'Connor, Symbolum Salacitatis, A Study of yhe God Priapus as a Literary Character, Frankfurt am Main, 1989, p. 67.

11. F. Cairns, op. cit., p. 207.

12. Voir Virgile, Buc., 10, 69 : Omnia uincit Amor et nos cedamus Amori ; Ovide, Am., 2, 18, 4 : (nos) et tener ausuros grandia frangit Amor ; 3, 11, 33-34: Luctantur pectusque leue in contraria tendunt/hac amor, hac odium, sed puto, uincit amor ; Cir., 437 : Omnia uincit Amor : quid enim non uinceret ille?

13. Pétrone s'est peut-être souvenu de ses vers dans la satire 87, où Eumolpe raconte comment il a séduit un jeune éphèbe: il a commencé par lui dérober des baisers qu'il payait ensuite de cadeaux; puis il a cessé ces complaisances; il a réussi alors à vaincre la résistance du jeune garçon, qui s'est transformée en ardeur, une fois que l'éphèbe a goûté au plaisir.

14. Je laisse ici de côté les interprétations à donner aux effets produits par l'inflexion bucolique sur la thématique élégiaque. Je m'intéresse seulement à ce que l'on peut en inférer sur les motifsclefs de l'élégie gallienne.

15. F. Cairns, op. cit., p. 174.

16. Je rejoins - en la reprenant dans la perspective d'une polémique - une hypothèse de M.P. Pieri à propos des renvois virgiliens dans l'élégie 1,4 (« Il dio Priapo in Tibullo I, $4:$ spunti bucolici di un elegiaco » dans Atti del Convegno Internazionale di Studi su Albio Tibullo, Roma, 1986, p. 88 : «E 
forse possibile supporre che in Tibullo non manchi la volontà di un sottile gioco allusiove con cui costringere il lettore al ricordo e al confronto con i recuperi vergiliani dal Thyrsis, improntati a «Stimmung-tragica »).

17. R. Thomas, op. cit., p. 57.

18. L'influence de l'élégie 1,4 est sensible chez les successeurs de Tibulle. Il n'est que de citer, par exemple, deux des élégies programmatiques de Properce, la 1,7 et la 1,8. l'élégie 1,7 lie expérience amoureuse et audience lectoriale : nos, ut consuemus, nostros agitamus amores/atque aliquid duram quaerimus in dominam/(...) me legat assidue post haec neglectus amator/et prosint illi cognita nostra mala (v. 5-6; 13-14). L'élégie I, 8, où les mots obsequio et tardo renvoient, sans doute, à Tibulle, corrige l'impression d'échec à laquelle pourrait conduire la précédente évocation des souffrances amoureuses : l'élégie a fondamentalement pour visée d'assurer la conquête de l'être aimé : hanc ego non auro, non Indis flectere conchis,/sed potui blandi carminis obsequio./Sunt igitur Musae neque amanti tardus Apollo,/quis ego fretus amo : Cynthia rara mea est (v. 39-42).

\section{AUTEUR}

JACQUELINE FABRE-SERRIS

Université Charles-de-Gaule - Lille 3 